\title{
Holographic system for copying holograms by using partially coherent light
}

\author{
I. Pascual, A. Beléndez, and A. Fimia
}

\begin{abstract}
A method for copying holographic optical elements with partially coherent light by using a holographic system is presented. The system includes two holographic lenses that are made as thick-phase holograms on silver halide-sensitized gelatin. The geometric conditions during reconstruction of the holographic system with white light are studied and the performance of the system is analyzed by a ray-tracing technique. The spatial and temporal coherence factors for the system are $>0.9$, and this indicates that it is possible to obtain good holographic elements with this holographic system. Copies of diffraction gratings and holographic lenses obtained with dichromated gelatin and photoresist as the recording material present good optical quality.
\end{abstract}

Key words: Holography, hologram copying, recording materials.

\section{Introduction}

For the past few years holographic technology and holographic optical elements (HOE's) have been developing quickly ${ }^{1}$ and optical designs that include holographic elements are beginning to appear in the literature. Optical systems containing scanners, ${ }^{2}$ head-up displays, ${ }^{3}$ waveguide couplers, ${ }^{4}$ and optical interconnectors ${ }^{5}$ have been developed thanks to holography. Holographic elements that belong to these optical systems need to have characteristics ${ }^{1}$ such as high diffraction efficiency, high signal-to-noise ratio (SNR), environmental stability, and transparency. Basically these characteristics are a function of the holographic recording medium and the chemical processing used in the fabrication of a HOE. ${ }^{6}$

The obtention of HOE's is limited when we want to record these HOE's by using a direct method with coherent light sources because the radiation emitted by the existent lasers does not correspond to the regions of high spectral sensitivity of suitable recording materials. A technique of solving this problem is to record the HOE's by means of a copy method. ${ }^{7}$ In this situation different wavelengths can be used in recording the master hologram and copying the final hologram. ${ }^{8}$ This solves the problem of the final material having little sensitivity at the desired replay

The authors are with the Departamento Interuniversitario de Optica, Laboratorio de Optica, Universidad de Alicante, Apartado 99, Alicante 03080, Spain.

Received 16 August 1991.

0003-6935/92/173312-08\$05.00/0.

(1) 1992 Optical Society of America. wavelength. Moreover, as a consequence of this, it is possible to work, for example, in the infrared region ${ }^{9}$ or in another spectral region.

Hologram copying has been studied extensively by Vanin ${ }^{10}$ and others, ${ }^{11-14}$ both in the Fresnel and in the Fraunhofer regions. However, in their studies coherent light was usually used for the copying. Likewise it is possible to copy holograms with partially coherent light.

The copying process consists of storing the interference pattern of the two wave fronts that are generated in the master hologram in a recording material with sufficient spatial resolution. Making copies is complicated and the difficulty lies in the precise recording of the spacing of the interference fringes that constitute the hologram element, as they are usually the size of micrometers.

In previous studies ${ }^{15}$ it has been shown that it is possible to copy HOE's by using partially coherent light sources whenever we place the master hologram in direct contact with the recording material we want to use to make the copy, with the photosensitive layers of the master and the copy placed together.

We describe a method for obtaining HOE mass production by using the hologram copying technique. We use partially coherent light and a holographic optical system to copy the holograms. Experimental results that demonstrate the feasibility of our method are presented. Finally we discuss these results.

\section{Experimental Setup}

The holographic optical system used is a thick-phase transmission holographic doublet that works as a 
holographic filter. ${ }^{16,17}$ The geometry of the system can be changed to select different wavelengths. Basically the holographic system is formed by two transmission holographic lenses with the same focal length separated by a distance that corresponds to twice the focal length of each of them, so that by working with white light we can select a particular wavelength that filters the image from the first lens on the focal plane by means of an appropriately sized diaphragm.

Let us consider the arrangement of the holographic system that uses two thick-phase transmission lenses, as shown in Fig. 1. The holographic lenses used in this system were constructed as in the setup shown in Fig. 2(a) and reconstructed as in the configuration shown in Fig. 2(b). We denote by $\mathrm{O}, \mathrm{R}, \mathrm{C}$, and $\mathrm{I}$ the sources that illuminate the object and reference light beams and the light beams that reconstruct and form the image, respectively. We make two waves interfere with each other, one being spherical and the other being collimated.

The recording material used was silver halidesensitized gelatin (SHSG) with the radiation of 633 $\mathrm{nm}$ of an $\mathrm{He}-\mathrm{Ne}$ laser. By this processing it is possible to obtain HOE's with high transmittance on the whole visible spectrum and at the same time an optimum SNR. ${ }^{18}$ HOE's present maximum diffraction efficiency near the theoretical value for the Bragg angle at this wavelength. The parameters corresponding to the two holographic lenses are shown in Table I.

The optical system presented can work for every wavelength emitted by the light source used. In this study the source used is a high-pressure mercury lamp. Table II shows the spectral response of the holographic system, taking into account the diffraction efficiency of the system.

The photosensitive materials used in this study for the manufacture of HOE's are sensitive to short wavelengths within the visible spectrum. For this reason the wavelength chosen for use in this study is $405 \mathrm{~nm}$. This wavelength and its corresponding bandwidth were selected with a $\mathrm{D}_{2}$ diaphragm (see Figure 1) that was suitable in terms of both size and position.

In order to select the $405-\mathrm{nm}$ wavelength we placed the $\mathrm{D}_{2}$ diaphragm in the focal plane of the first $\mathrm{HOE}$ corresponding to $405 \mathrm{~nm}$. When a collimated beam of white light is incident on lens $L_{1}$ it is possible to focus the whole set of wavelengths $\lambda$ along one line. By filtering $\lambda$ adequately it is possible to use a divergent

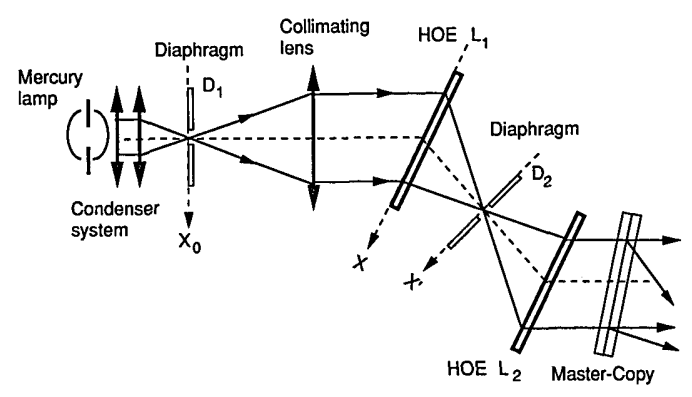

Fig. 1. Holographic system setup.

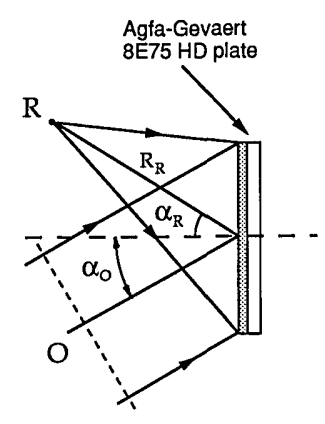

(a)

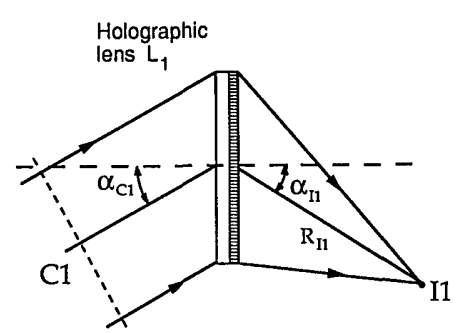

(b)

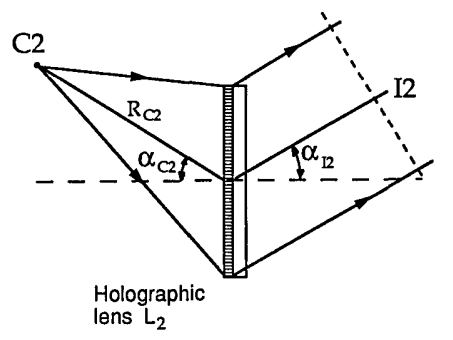

Fig. 2. Hologram construction (a) and reconstruction (b) geometry.

beam so that the second lens serves as a collimator. Thus we can select the wavelength and its bandwidth by choosing the relative angle of the system in relation to the entrance beam and the position and size of the $\mathrm{D}_{2}$ diaphragm.

The coherence factor of this system is determined by the angular size of the source and in our case by the diameter of the opening of the filter and the focal point of lens $\mathrm{L}_{2}$. Therefore it is important to know the shape of the beam used in the copy as well as the shape and size of the focalization region of the first HOE. This information is fundamental because the temporal coherence factor is related to the wavelengths that cross the opening that is used as a source for the second HOE.

The behavior of a holographic optical system can be studied thanks to the development of all the topics related to the recording and reconstruction geome-

Table I. Parameters for Two Holographic Lenses at a 633-nm Wavelength

\begin{tabular}{ll}
\hline \multicolumn{1}{c}{ Parameter } & Measurement \\
\hline Exit pupil diameter $(D)$ & $100 \mathrm{~mm}$ \\
Focal length $(f)$ & $200 \mathrm{~mm}$ \\
Aperture $(f / D)$ & $f / 2$ \\
Carrying frequency & 1000 lines $/ \mathrm{mm}$ \\
\hline
\end{tabular}


Table II. Spectral Response of the Holographic System

\begin{tabular}{ccc}
\hline $\begin{array}{c}\text { Maximum Diffraction } \\
(\mathrm{nm})\end{array}$ & $\begin{array}{c}\text { Efficiency for Each Lens } \\
(\%)\end{array}$ & $\begin{array}{c}\text { Maximum Diffraction } \\
\text { Efficiency for the Holographic } \\
\text { System Doublet (\%) }\end{array}$ \\
\hline 405 & 61 & 38 \\
436 & 61 & 38 \\
488 & 60 & 37 \\
514 & 60 & 37 \\
546 & 47 & 22 \\
578 & 48 & 23 \\
633 & 45 & 20 \\
\hline
\end{tabular}

tries of HOE's, their functions, the range of wavelengths in which they are going to function, and the possible aberrations that can appear. Many authors ${ }^{19-25}$ work in this research area of holographic optics. All of them study the geometric relationships that exist between the coordinates of the object, reference, reconstruction, and image sources, and obtain the mathematical equations for the aberrations that appear in the reconstructed beam image. These analytical equations for the aberrations provide information on the quality of the images in HOE's. Nevertheless other techniques that also allow for the analysis of the quality of these holographic systems exist. They have become absolutely necessary, especially if the element must work with a convergent beam image as is the case of the holographic system we are considering in our study. We are referring here to the techniques based on ray tracing.

This ray-tracing technique in HOE's has been analyzed by many authors for more than twenty years and analytic equations have been developed to carry out this tracing. ${ }^{26-30}$ The need to have a raytracing method for analyzing HOE's has prompted us to write a computer program for use in ray tracing in HOE's in order to obtain the greatest amount of information possible from them. This ray-tracing method is used with volume HOE's only (diffracted order +1 ), and by using this method it is possible to obtain the direction cosines of the diffracted rays through the HOE as well as the points of intersection of these rays with an arbitrary plane. By making this arbitrary plane coincide with the plane that is perpendicular to the main ray that passes through the Gaussian point information about the size of the spot on the image plane can be obtained. On the other hand it is possible to obtain information about the quality of the images produced by the HOE's by applying the rms concept. ${ }^{30,31}$ Likewise the raytracing method used allows us to analyze any type of configuration of two HOE's as long as we know their obtention and reconstruction geometries. ${ }^{30}$

In ray tracing the image plane is characterized by coordinates $x^{\prime}$ and $y^{\prime}$. These coordinates correspond to a plane that includes the image focal point of lens $\mathrm{L}_{1}$ for $\lambda=405 \mathrm{~nm}$ and that is perpendicular to the $O Z^{\prime}$ direction, as is seen in Fig. 3. This $O Z^{\prime}$ direction is determined by the ray that emerges from the center

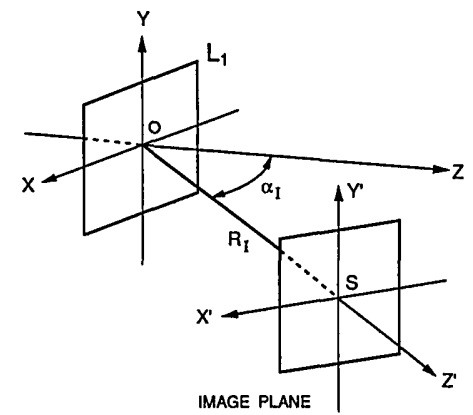

Fig. 3. Scheme of the focalization plane $(405 \mathrm{~nm})$ through $\mathrm{L}_{1}$.

of $\mathrm{L}_{1}$ and passes through its Gaussian image focal point for the wavelength being used. The $\mathrm{O}^{\prime}$ origin of the system of coordinates $X^{\prime} Y^{\prime} Z^{\prime}$ coincides with this Gaussian image point, which is denoted by $S$ in Fig. 3 .

In order to study the shape and size of the spot that corresponds to the area of focalization for $\lambda=405 \mathrm{~nm}$ a beam formed by a group of rays parallel to the optical axis of the holographic lens $L_{1}$ was used. These parallel rays were distributed over the lens, as shown in Fig. 4, covering a diameter of $9.5 \mathrm{~cm}$. They are incident to $L_{1}$ with Bragg's angle, which allows for maximum diffraction efficiency for this wavelength.

At the outset the ray tracing is done while assuming that the source of illumination is a point. The shape and size of the spot on the studied plane for this situation is shown in Fig. 5, in which the wavelength utilized is $405 \mathrm{~nm}$. In Fig. 6 we can see the shape and size of the spectrum when the holographic system is illuminated under the same conditions but with white light.

In our second analysis, we again studied the behavior of the first holographic element $L_{1}$ when it was illuminated by an extended source. The plane that interests us continues to be the $X^{\prime} Y^{\prime}$ plane of Fig. 3 and this area of interest corresponds to the focalization zone for a wavelength of $405 \mathrm{~nm}$. The size of the source $\mathrm{D}_{1}$ was $2 \mathrm{~mm}$ in diameter and the calculations that were done by using the ray-tracing program were carried out for three point sources in the $X_{0}$ direction, perpendicularly to the optical axis of the system that is contained in the plane of the scheme shown in Fig.

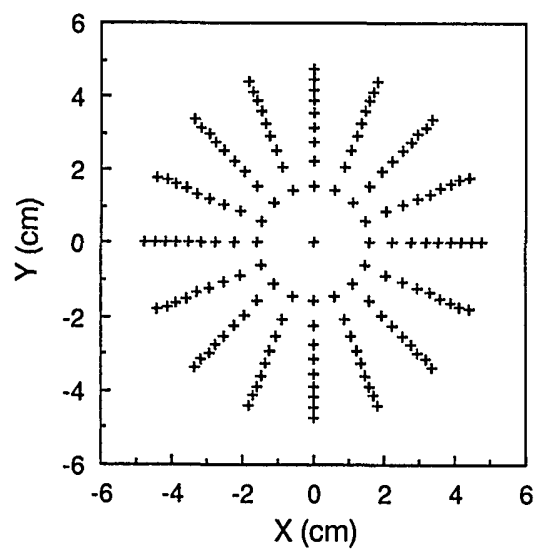

Fig. 4. Rays in the entrance pupil of $\mathrm{L}_{1}$ for numerical ray tracing. 


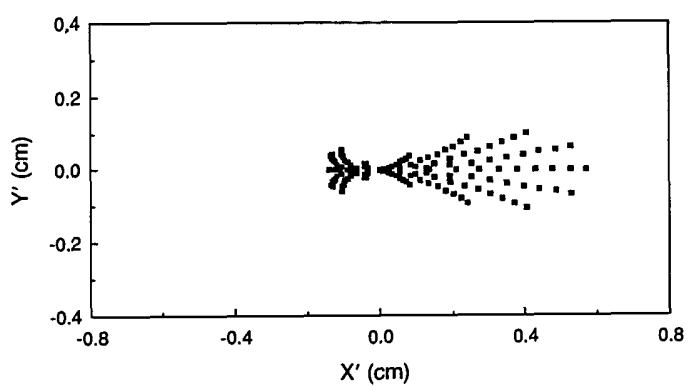

Fig. 5. Calculated spot diagram on the $X^{\prime} Y^{\prime}$ plane for $405 \mathrm{~nm}$ and punctual light source.

5 , and three point sources in the $Y_{0}$ direction, perpendicularly to the optical axis of the system and perpendicularly to the plane of the scheme in the same figure. In each direction a point source was used that was situated in the center of the $D_{1}$ opening, another that was situated $1 \mathrm{~mm}$ above the central one, and finally, another that was situated underneath. The same was done in the other direction so that the two central points were made to coincide, thus providing five point sources for the study. The size and shape of the spot are shown in Fig. 7.

By placing a $D_{2}$ diaphragm on the study's image plane a second extended source of illumination is obtained that provides radiation with a bandwidth of $10 \mathrm{~nm}$, measured with a spectrophotometer, and centered at $405 \mathrm{~nm}$, which will illuminate the optical system's second HOE $\mathrm{L}_{2}$. The bandwidth of this radiation as well as the angular size of the light source determine the coherence conditions of our optical system and then condition the copying process that is to be carried out.

In order to complete the information on the aberrations of the holographic system used in this study the rms, $\sigma\left(r_{i}{ }^{\prime}\right)$, is determined:

$$
\sigma\left(r_{i}^{\prime}\right)=\left\{\frac{1}{N} \sum_{i=1}^{N}\left[\left(X_{i}^{\prime}-\left\langle X^{\prime}\right\rangle\right)^{2}+\left(Y_{i}^{\prime}-\left\langle Y^{\prime}\right\rangle\right)^{2}\right]\right\}^{1 / 2},
$$

where $\left(X_{i}^{\prime}, Y_{i}^{\prime}\right)$ are the coordinates of an arbitrary point corresponding to the intersection of the ray that emerges from point $\left(X_{i}, Y_{i}\right)$ of the exit pupil of $\mathrm{L}_{1}$ and the plane of analysis $r_{i}^{\prime}=\left(X_{i}^{\prime 2}+Y_{i}^{\prime 2}\right)^{1 / 2}$ and we use $\left\langle X^{\prime}\right\rangle$ and $\left\langle Y^{\prime}\right\rangle$ to designate the coordinates of the geometric center of this distribution of points on the plane (Fig. 8).

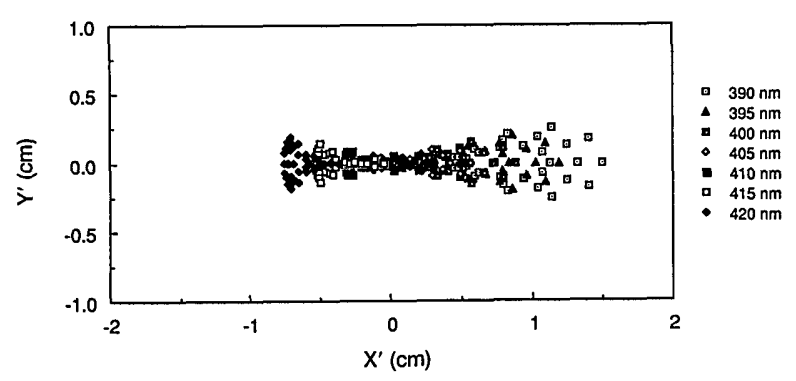

Fig. 6. Calculated spot diagram on $X^{\prime} Y^{\prime}$ plane for white light and punctual light source.

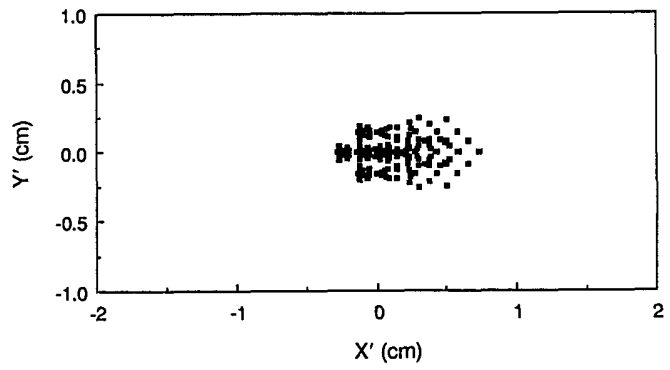

Fig. 7. Calculated spot diagram on $X^{\prime} Y^{\prime}$ plane for $405 \mathrm{~nm}$ and extended light source.

As can be seen, the holographic system presents astigmatism, coma and spherical aberrations (Fig. 5). Nevertheless the image point can be said to be equivalent to a circle with a radius of $0.196 \mathrm{~cm}$, with the value of the center ${ }^{31}$ of this circle being the average of the point distribution $\left(\left\langle X^{\prime}\right\rangle=0.082 \mathrm{~cm}\right.$ and $\left.\left\langle Y^{\prime}\right\rangle=0 \mathrm{~cm}\right)$ and the radius being equal to the $\mathrm{rms}$ $\sigma\left(r_{i}^{\prime}\right)$. This circle contains $75 \%$ of all the rays that emerge from $\mathrm{L}_{1}$. These circumstances allow us to maximize light use and minimize loss.

Finally we compared the system's incident beam with the emerging beam. In Fig. 9 the $P$ and $\mathrm{P}^{\prime}$ sections that we wish to compare are shown. $P$ corresponds to a section of the ray beam that is incident to the system and $\mathrm{P}^{\prime}$ corresponds to a section of the ray beam that emerges from the system. Both sections $P$ and $P^{\prime}$ have been included in the same system of coordinates while noting the presence of astigmatism caused by the shape of the set of rays that emerges.

Given that HOE's are going to be made through a copying process, the original HOE must have a series of qualities such as high transmittance for any wavelength, light stability, panchromatic response, low noise levels, the behavior of a volume element, a 1:1 relation between the diffraction order and the transmitted order, and, lastly, environmental stability. The photochemical processing that allows us to obtain these characteristics is SHSG, as we mentioned above. The master we produced was a 1000 -lines $/ \mathrm{mm}$ diffraction grating with the energy to produce a $1: 1$ ratio in the reconstruction step by using a wavelength of $405 \mathrm{~nm}$. Likewise a holographic lens was made with a $165-\mathrm{mm}$ focal point at $405 \mathrm{~nm}$ whose diffraction efficiency is uniform over the entire exit pupil of

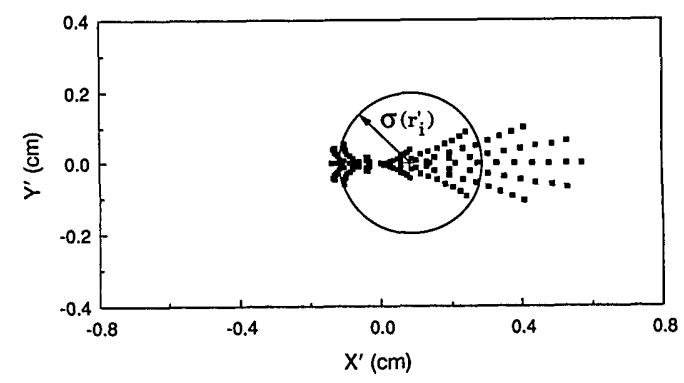

Fig. 8. $\operatorname{rms~} \sigma\left(r_{i}{ }^{\prime}\right)=0.196 \mathrm{~cm}$ on the $X^{\prime} Y^{\prime}$ plane for $405 \mathrm{~nm}$ and a punctual light source. 


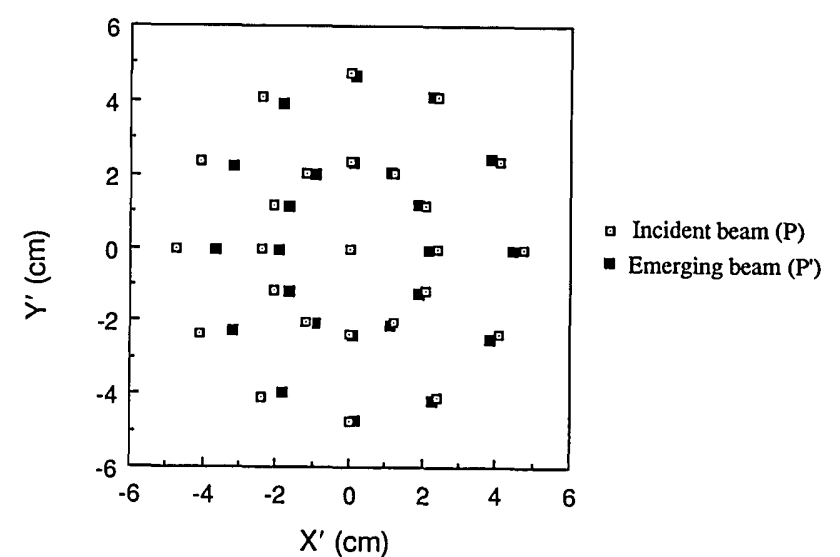

Fig. 9. Comparison of the holographic system's emergent and incident beams.

the system. The diameter of the lens used as a master was $60 \mathrm{~mm}$. Both masters were produced with a wavelength of $633 \mathrm{~nm}$ by a $15-\mathrm{mW} \mathrm{He}-\mathrm{Ne}$ laser. Transmission reached $80 \%$.

\section{Coherence Conditions}

All light sources are characterized by a coherence factor that, in some way, defines the quality of each source. ${ }^{32}$ The total coherence factor of a source can be expressed, except in the case of constant phase factors, as a product of the spatial coherence factor $\Gamma$, which is associated with the angular size of the source, and the temporal coherence factor $K$, which is associated with the spectral bandwidth emitted by the source. From the point of view of theoretical calculations, the spatial coherence factor $\Gamma$ is obtained by using the Van Citter-Zernike theorem. For a circular source with an angular size of $\gamma$, which is shown in Figure 10, and a uniform intensity distribution, $\Gamma$ is derived from the normalized first-order Bessel function:

$$
\Gamma=\frac{2 J_{1}(v)}{v},
$$

where

$$
v=2 \pi \gamma r / \lambda, \quad r=\left(h_{1}+a+h_{2}\right) \sin \theta^{\prime},
$$

where $\gamma$ is the angular size of the source, $\lambda$ is the working wavelength, $h_{1}$ is the thickness of the master, $h_{2}$ is the thickness of the copy, $a$ is the thickness

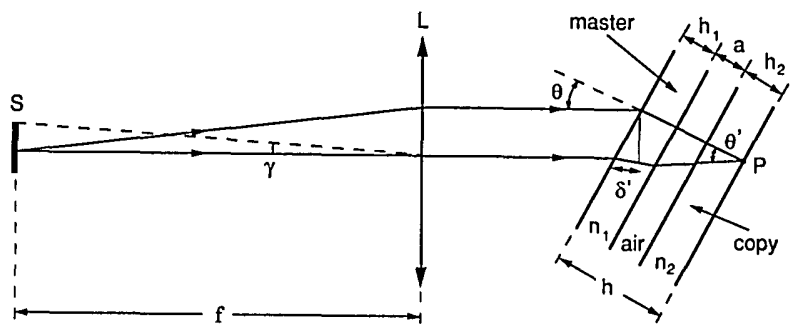

Fig. 10. Scheme of the optical path of the rays that interfere in the copying process.

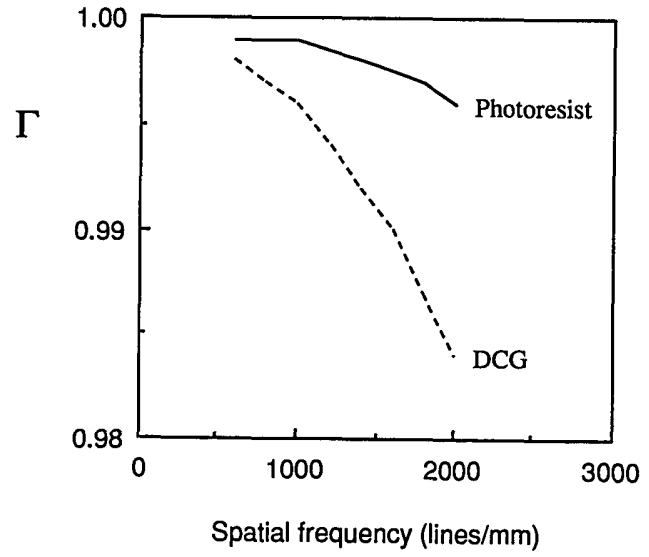

Fig. 11. Spatial coherence versus spatial frequency for photoresist and DCG.

of the layer of air between the two photosensitive layers when they are joined, and $\theta^{\prime}$ is the Bragg angle in the interior of the photosensitive medium. These parameters are shown in Fig. 10. The temporal coherence factor $K$ is determined by the spectral bandwidth of the radiation emitted by the source. The corresponding calculations are included in the bibliography $^{33}$ for cases in which the thermic emission source has a Gaussian profile and the equation is

$$
K=\exp \left[-\pi\left(-\Delta \lambda / \lambda^{2}\right) \delta^{\prime}\right]^{2},
$$

where $\delta^{\prime}$ is the difference of the optical path from the source to the point where the two beams intersect, $\Delta \lambda$ is the bandwidth of the emitted radiation, and $\lambda$ is the wavelength used in the study. Likewise the difference in the optical path $\delta^{\prime}$ can be expressed as a function of the refraction indexes and the thicknesses of the recording materials used $n_{1}, n_{2}, h_{1}, h_{2}$, as well as of the Bragg angle $\theta$ y $\theta^{\prime}$ inside and outside the recording material for maximum efficiency ${ }^{34}$ :

$$
\delta^{\prime}=h_{1} n_{1}\left(1-\cos \theta^{\prime}\right)+h_{2} n_{2}\left(1-\cos \theta^{\prime}\right)+a(1-\cos \theta) .
$$

Parameter $a$ represents the thickness of the air layer that exists between the two layers of photosensi-

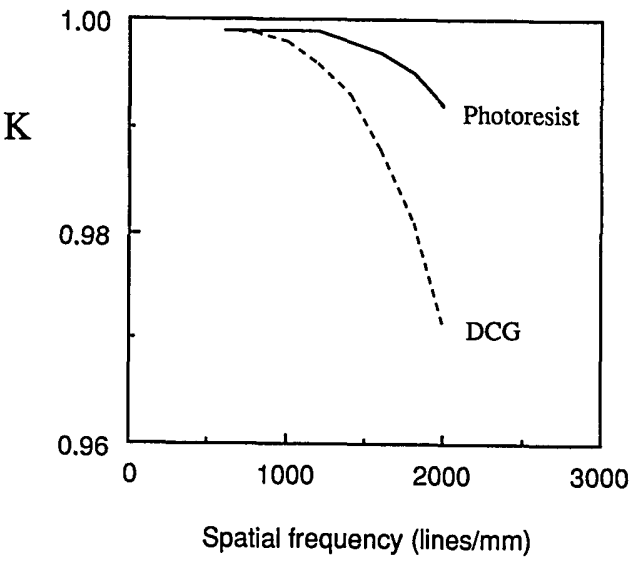

Fig. 12. Temporal coherence versus spatial frequency for photoresist and DCG. 


\begin{tabular}{|c|c|c|}
\hline Step & Process & Time \\
\hline \multirow[t]{5}{*}{ Preparation of plates } & 1. Soak in fixer (done in dark) & $15 \mathrm{~min}$ \\
\hline & 2. Rinse in running water (done in dark) & $15 \mathrm{~min}$ \\
\hline & 3. Rinse in methyl alcohol & $10 \mathrm{~min}$ \\
\hline & 4. Rinse in clean methyl alcohol & $10 \mathrm{~min}$ \\
\hline & 5. Dry in vertical position & $>6 \mathrm{~h}$ \\
\hline \multirow[t]{5}{*}{ Sensitizing plates (done in red light) } & 6. Soak in ammonium dichromate in flat pan (emulsion side up) & $5 \mathrm{~min}$ \\
\hline & 7. Tilt plate $\sim 10^{\circ}$ and allow excess to run off & $3 \mathrm{~min}$ \\
\hline & 8. Remove residue at edge with a paper towel & \\
\hline & 9. Store in light tight box with same tilt until ready to use & \\
\hline & 10. Dry in ambiental conditions & $15-40 \mathrm{~h}$ \\
\hline \multirow{5}{*}{ Developing plates } & 11. Rinse in running water (done in dark) & $20 \mathrm{~min}$ \\
\hline & 12. Dehydrate in $50 \%$ isopropanol & $3 \mathrm{~min}$ \\
\hline & 13. Dehydrate in $90 \%$ isopropanol & $3 \mathrm{~min}$ \\
\hline & 14. Dehydrate in $100 \%$ isopropanol & $3 \mathrm{~min}$ \\
\hline & 15. Dry by baking at $65^{\circ} \mathrm{C}$ & $20 \mathrm{~min}$ \\
\hline
\end{tabular}

${ }^{a}$ Fixer formula is $10 \% \mathrm{Na}_{2} \mathrm{~S}_{2} \mathrm{O}_{3} \cdot 5 \mathrm{H}_{2} \mathrm{O}$, sensitizing formula is $0.5 \%$ ammonium dichromate, and plates are Kodak $649 \mathrm{~F}$.

tive material when the copy is made. The value of parameter $a$, in our experience, has been $3 \mu \mathrm{m}$ and the measurements of this parameter have been done by interferential methods. ${ }^{35}$

In Figs. 11 and 12 the spatial and temporal coherence factors have been represented as they relate to spatial frequency. The spatial frequency used ranged from 500 to 2000 lines $/ \mathrm{mm}$ as this is the range of variation in spatial frequency that the original lenses present, and therefore it is the range of the copies to be made. In these figures we can see the variation of said factors if we take into account the recording materials used in the copies: dichromated gelatin (DCG) with a 15- $\mu \mathrm{m}$ thickness and photoresist with a $1-\mu \mathrm{m}$ thickness, and the variation when the angular size of the source $\gamma$ is increased and the bandwidth used is $\Delta \lambda$. In any case the values of the coherence factors are greater than 0.9 and there is a decrease in the spatial coherence factor only when the source has a large angular size and the spatial frequency is 2000 lines $/ \mathrm{mm}$. Therefore we can conclude that if the coherence conditions of a holographic system are taken into account it is possible to obtain copies that have good diffraction efficiency.

Table IV. Photoresist Processing Schedule

\begin{tabular}{|c|c|c|}
\hline Step & Process & Time \\
\hline $\begin{array}{l}\text { 1. Method for obtaining photoresist layers } \\
\text { 1.1. Cleaning the glass plate }\end{array}$ & $\begin{array}{l}\text { Bathe in saturated soda lye } \\
\text { Wash in running water } \\
\text { Bathe in chromic compound } \\
\text { Wash in running water } \\
\text { Wash in distilled water } \\
\text { Dry in } 120^{\circ} \mathrm{C} \text { oven } \\
\text { Let cool in clean atmosphere }\end{array}$ & $\begin{array}{l}20 \mathrm{~min} \\
20 \mathrm{~min} \\
24 \mathrm{~h} \\
20 \mathrm{~min} \\
5 \mathrm{~min} \\
20 \mathrm{~min}\end{array}$ \\
\hline $\begin{array}{l}\text { 1.2. Emulsion process (done in red light) } \\
\text { 1.3. Drying the layers (process done in red light) }\end{array}$ & $\begin{array}{l}\text { Compound of } 65 \% \mathrm{AZ}-1350 \mathrm{~J} \text { Shipley and } 35 \% \mathrm{AZ} \text { - } \\
1350 \mathrm{~B} \text { Shipley, both filtered } \\
\text { Centrifuge at } 2000 \mathrm{rev} / \mathrm{min} \\
\text { Dry in } 80^{\circ} \mathrm{C} \text { oven }\end{array}$ & $\begin{array}{l}45 \mathrm{~s} \\
20 \mathrm{~min}\end{array}$ \\
\hline 2. Processing (red light) & $\begin{array}{l}\text { AZ-303 developer at } 20^{\circ} \mathrm{C} \\
\text { Wash in distilled water } \\
\text { Dry in } 80^{\circ} \mathrm{C} \text { oven }\end{array}$ & $\begin{array}{l}45 \mathrm{~s} \text { (agitation) } \\
5 \mathrm{~min} \\
20 \mathrm{~min}\end{array}$ \\
\hline 3. Bathing compounds & & Amount \\
\hline 3.1. Saturated soda lye & $\begin{array}{l}\text { Sodium hydroxide } \\
\text { Tap water }\end{array}$ & saturated \\
\hline 3.2. Chromic compound & $\begin{array}{l}\text { Sulphuric acid } \\
\text { Potassium dichromate } \\
\text { Tap water }\end{array}$ & $\begin{array}{l}80 \mathrm{ml} \\
20 \mathrm{~g} \\
20 \mathrm{ml}\end{array}$ \\
\hline 3.3. AZ-303 & $\begin{array}{l}\text { Shipley's } \\
\text { commercial developer }\end{array}$ & $5 \%$ in volume \\
\hline
\end{tabular}

${ }^{a}$ For a $10 \mathrm{~cm} \times 10 \mathrm{~cm}$ glass plate, $2 \mathrm{~g}$ of the compound are used. 


\section{Experiments and Results}

Experiments were carried out with DCG from a Kodak $649 \mathrm{~F}$ emulsion and positive photoresist from Shipley AZ-1350 J and B. DCG ${ }^{36}$ is a gelatin layer that contains a small amount of dichromate; it has large refractive index modulation capability, high resolution, and low absorption and scattering. With this material a volume-phase hologram is formed. On the other hand photoresists ${ }^{37}$ are light-sensitive organic films that yield a relief image after exposure and development. With this material a thin-phase hologram is formed. It has no grain structure so scattered light is negligible. In these experiments a collimated beam from a high-pressure mercury lamp $(\lambda=405$ $\mathrm{nm}$ ), which was incident at Bragg angle to the master and copy, was used to produce a series of transmission gratings. Gratings were recorded with exposures ranging from 10 to $120 \mathrm{~mJ} / \mathrm{cm}^{2}$. The exposed plates were developed. Details of the processing schedule are given in Tables III ${ }^{36}$ and IV. ${ }^{37}$ Measurements of the diffraction efficiency of these gratings at the Bragg angle were made. The diffraction efficiency was defined as $\eta=I_{1} / I_{0}$ where $I_{1}$ was the power in the first diffracted order and $I_{0}$ was the power transmitted by an unexposed area on the plate, i.e., the incident power less losses caused by absorption and reflection at the two surfaces.

Curves showing the variation of the peak diffraction efficiency with exposure are presented in Figs. 13 and 14. The master we used had a spatial frequency of 1000 lines $/ \mathrm{mm}$. As can be seen in Fig. 13 the diffraction efficiency obtained with a partially coherent light copy has a maximum value of $85 \%$, which is similar to those obtained with coherent light. In Fig. 14, with photoresist the grating obtained with copy has a lower maximum value of $40 \%$ in diffraction efficiency. This fall in diffraction efficiency is due to the type of recorded grating. Gratings obtained in DCG are thick gratings whereas gratings obtained in photoresist are thin gratings, and with plane gratings it is not possible to obtain a diffraction efficiency of more than $33 \%$ when working in linear conditions.

However the important thing is that it is possible to obtain gratings with partially coherent light from a holographic system in DCG and photoresist that are similar to those obtained with coherent light. This

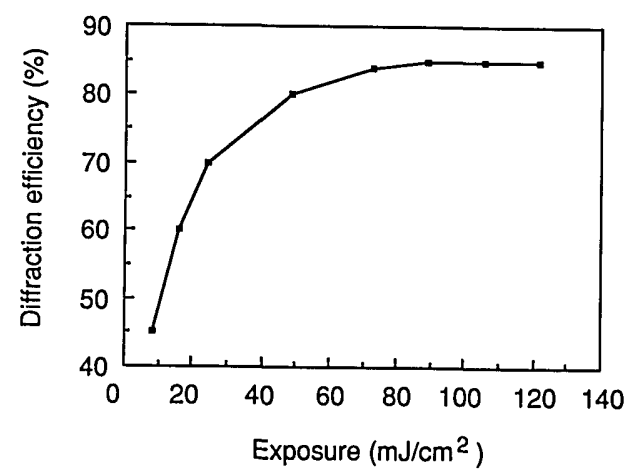

Fig. 13. Diffraction efficiency versus exposure with DCG. The spatial frequency of the master hologram is 1000 lines $/ \mathrm{mm}$.

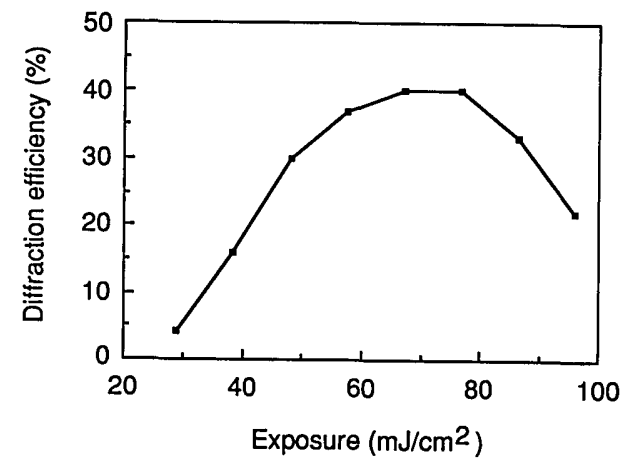

Fig. 14. Diffraction efficiency versus exposure with photoresist. The spatial frequency of the master hologram is 1000 lines $/ \mathrm{mm}$.

allows us to work in a region of the spectrum in which no laser exists.

It is important to point out that we have obtained holographic lenses with partially coherent light from a holographic system in DCG and a photoresist 50 $\mathrm{mm}$ in diameter.

\section{Conclusions}

A method for copying with partially coherent light by using a holographic system that allows the selection of different wavelengths of the source spectrum by the position and size of the aperture stop has been analyzed experimentally, and diffraction gratings have been obtained by using DCG and photoresist, both of which have a high degree of diffraction efficiency. The coherence conditions used are suitable for copying all types of transmission HOE's in any recording material with optimum results that are similar to those obtained by using direct methods. A method for the production of series HOE's by holographic optics has also been described. And finally, it is important to point out that the holographic optical system used to make copies has great stability and allows for long exposures of up to $\sim 3 \mathrm{~h}$.

\section{References}

1. G. T. Sincerbox, "Challenges for the use of holographic elements in optical storage," in Holographic Optics II: Principles and Applications, G. M. Morris, ed., Proc. Soc. Photo-Opt. Instrum. Eng. 1136, 80-91 (1989).

2. G. T. Sincerbox, "Holographic scanners: applications, performance and design," in Laser Beam Scanning, G. F. Marshall, ed. (Dekker, New York, 1985), pp. 1-62.

3. D. W. Swift, "The application of holographic optical elements to head-up displays," in Proc. Inst. Radio Electron. Eng. Austr. 76, 93-97 (1987).

4. O. D. D. Soares, "Holographic coupler for fiber optics," Opt. Eng. 20, 740-745 (1981).

5. J. W. Goodman, F. Leonberger, S. Y. Kung, and R. Athale, "Optical interconnections for VLSI systems," Proc. IEEE 72, 850-866 (1984).

6. P. Hariharan, Optical Holography (Cambridge U. Press, London, 1984), Chaps. 6 and 7.

7. W. T. Rhodes, "Hologram copying," in Handbook of Optical Holograpy, H. J. Cauldfield, ed. (Academic, New York, 1979), pp. 373-378. 
8. L. H. Lin and E. T. Doherty, "Efficient and aberration-free wavelength reconstruction from holograms illuminated at wavelengths differing from the forming wavelength," Appl. Opt. 10, 1314-1318 (1971).

9. H. P. Herzig, "Holographic optical elements (HOE) for semiconductor laser," Opt. Commun. 58, 144-148 (1986).

10. V. A. Vanin, "Hologram copying (review)," Sov. J. Quantum Electron. 8, 809-818 (1978).

11. P. Hariharan, Optical Holography (Cambridge U. Press, London, 1984), Chap. 1.

12. D. B. Brumm, "Copying holograms," Appl. Opt. 5, 1946-1947 (1966).

13. M. J. Landry, "The effects of two hologram-copying parameters on the qualities of copies," Appl. Opt. 6, 1947-1956 (1967).

14. J. C. Palais and J. A. Wise, "Improving the efficiency of very low efficiency holograms by copying," Appl. Opt. 10, 667-668 (1971).

15. N. J. Phillips and D. Martens, "Aspects of the copying of holograms using incoherent light," in Progress in Holographic Applications, J. P. Ebbeni, ed., Proc. Soc. Photo-Opt. Instrum. Eng. 600, 123-126 (1985).

16. A. Beléndez, I. Pascual, and A. Fimia, "Imaging in white light with a thick-phase transmission holographic doublet," J. Opt. 20, 263-268 (1989).

17. I. Pascual, A. Beléndez, and A. Fimia, "Holographic optical system to copy holographic optical elements," in Holographic Optics III: Principles and Applications, G. M. Morris, ed., Proc. Soc. Photo-Opt. Instrum. Eng. 1507, 373-378 (1991).

18. A. Fimia, A. Beléndez, and I. Pascual, "Silver halide (sensitized) gelatin in Agfa-Gevaert plates: the optimized procedure," J. Mod. Opt. 38, 2043-2051 (1991).

19. R. W. Meier, "Magnification and third-order aberrations in holography," J. Opt. Soc. Am. 55, 987-992 (1965).

20. E. B. Champagne, "Nonparaxial imaging, magnification and aberration properties in holography," J. Opt. Soc. Am. 57, 51-55 (1967).

21. J. N. Latta, "Computer-based analysis of hologram imagery and aberrations I: Hologram types and their nonchromatic aberrations," Appl. Opt. 10, 599-608 (1971).

22. K. Winick, "Designing efficient aberration-free holographic lens in the presence of a construction-reconstruction wavelength shift,"'J. Opt. Soc. Am. 72, 143-148 (1982).
23. H. Chen, R. H. Hershey, and E. N. Leith, "Design of a holographic lens for the infrared," Appl. Opt. 26, 1983-1988 (1987).

24. Y. Amitai and A. A. Friesem, "Combining low aberrations and high diffraction efficiency in holographic optical elements," Opt. Lett. 13, 883-885 (1988).

25. A. Beléndez, I. Pascual, and A. Fimia, "Influences of recording materials in HOE," in Holographic Optics II: Principles and Applications, G. M. Morris, ed., Proc. Soc. Photo-Opt. Instrum. Eng. 1136, 58-65 (1989).

26. I. A. Abramowitz, "Evaluation of hologram imaging by raytracing," Appl. Opt. 8, 403-410 (1969).

27. J. N. Latta, "Computer-based analysis of holography using ray-tracing," Appl. Opt. 10, 2698-2710 (1971).

28. W. T. Weldford, "A vector ray-tracing equation for hologram lenses of arbitrary shape," Opt. Commun. 14, 322-323 (1975).

29. H. W. Holloway and R. A. Ferrante, "Computer analysis of holographic systems by means of vector ray tracing," Appl. Opt. 20, 2081-2084 (1981).

30. A. Beléndez, I. Pascual, and A. Fimia, "Computer analysis of holographic optical elements using ray tracing," Opt. Pure Appl. 24, 135-153 (1991).

31. Y. Ishii and K. Murata, "Reflection holographic lens having nonspherical wavefront designed by a microcomputer," Opt. Commun. 47, 303-308 (1983).

32. M. Born and E. Wolf, Principles of Optics, 6th ed. (Pergamon, Oxford, 1987), Chap. 10, pp. 491-513.

33. H. H. Hopkins, "The theory of coherence and its applications," in Advanced Optical Techniques, A. C. S. Van Heel, ed. (North-Holland, Amsterdam, 1967), p. 232.

34. I. Pascual, "Obtención de elementos opticos holográficos con luz parcialmente coherente mediante la utilización de un sistema holográfico," Ph. D. dissertation (University of Valencia, Valencia, Spain, 1990).

35. J. M. Altman, "Method for measuring the absolute separation between glass plates during contact printing," Photogr. Sci. Eng. 14, 428-431 (1970).

36. D. Meyerhofer, "Dichromated gelatin," in Holographic Recording Materials, Vol. 20 of Topics in Applied Physics, H. M. Smith, ed. (Springer-Verlag, Berlin, 1977), pp. 75-99.

37. R. A. Bartolini, "Photoresist" in Holographic Recording Materials, Vol. 20 of Topics in Applied Physics, H. M. Smith, ed. (Springer-Verlag, Berlin, 1977), pp. 209-227. 